\title{
Punctal occlusion in Sjögren's syndrome needs clarification
}

Eduardo M. Rocha, Jayter S. Paula and Peter S. Reinach

The July 2012 issue of Nature Reviews Rheumatology included a Review article by Ramos-Casals et al. (Topical and systemic medications for the treatment of primary Sjögren's syndrome. Nat. Rev. Rheumatol. 8,399-411;2012), ${ }^{1}$ which comprehensively reviewed the therapeutic options available to treat Sjögren's syndrome (SS). In this article, the authors indicate that lacrimal punctal occlusion is a viable therapeutic option; however, none of the references that they cited clearly support this conclusion. Furthermore, the results of studies not cited in the article indicate that this procedure might not be a viable treatment for the symptoms of SS.

In Figure 1 of their article, RamosCasals and co-authors assign a grade of $1 \mathrm{~B}$ to punctual occlusion efficacy, based on a scale developed by Harbour and Miller. ${ }^{2} \mathrm{~A}$ grading of $1 \mathrm{~B}$ is reserved for evidence from high quality systematic reviews examining studies that adhered to case-control or cohort paradigms. Thus, this grade implies that the recommendation is formed on the basis of information from correctly conducted meta-analyses of data from systematic reviews of randomized controlled trials (RCTs), or from RCTs with a low risk of bias support. Assigning the grade $1 \mathrm{~B}$ to punctal occlusion is ill-founded, as the three references cited by Ramos-Casals and colleagues to support this recommendation do not provide the evidence needed to justify such a grading. The first of these citations is a nonsystematic review article by Fox ${ }^{3}$ wherein punctal occlusion is merely listed in a table as a therapeutic option, with no data provided regarding its therapeutic efficacy. The second reference is a descriptive review in which punctal occlusion strategy is only mentioned in a description of a treatment session; ${ }^{4}$ again, no data or conclusions are provided regarding the usefulness of punctal occlusion. Finally, the third reference cited in support of the $1 \mathrm{~B}$ grading of punctal occlusion is, in fact, a systematic review, ${ }^{5}$ but only one interventional study that provides data supporting punctal occlusion effectiveness is examined therein. ${ }^{6}$ Moreover, this interventional study is of questionable value as it describes an open label study that included just 30 patients (10 per treatment group) who were followed for just 6 months. ${ }^{6}$ Concerns exist regarding the inclusion of this interventional study in the systematic review because: a) it is unclear if the patients enrolled had SS; b) no control group was assigned; and c) artificial tears treatment regimen was not controlled for among the participants of the study., ${ }^{5,6}$

Another consideration that severely detracts from favourable evaluation of punctual occlusion efficacy is data from other clinical studies suggesting, conversely, that punctal occlusion is not useful in dry eye syndrome. Patients with lacrimal drainage system obstruction caused by chronic dacryocystitis, a condition that mimics the effect of punctual occlusion, have reduced tear secretion compared with control individuals. ${ }^{7}$ This observation suggests that a better option would be to perform dacryocystorhinostomy -that is, re-opening of the lacrimal drainage system-to increase tear production. ${ }^{8}$ Furthermore, in healthy individuals, insertion of a punctal plug caused secretion to decline rather than increase. ${ }^{9}$ Moreover punctal occlusion does not improve the comfort of patients with dry eye disease, as revealed by a double-blind study. ${ }^{10}$

On the basis of this evidence, a cautionary note should be taken when considering the conclusion, at this point, that punctal occlusion improves ocular surface hydration in patients with SS. In our opinion, Ramos-Casals et al. ${ }^{1}$ are mistaken in giving this procedure such a strong recommendation by assigning a grade of 1B. Further evidence from double-blind RCTs is needed before punctal occlusion can be recommended to treat ocular surface dryness found in patients with SS.

Department of Ophthalmology,

Otorhinolaryngology and Head and Neck

Surgery, FMRP, São Paulo University, Avenue

Bandeirantes, 3900, 14049-900 Ribeirao

Preto, São Paulo, Brazil (E. M. Rocha,

J. S. Paula, P. S. Reinach).

Correspondence to: E. M. Rocha

emrocha@fmrp.usp.br

doi:10.1038/nrrheum.2012.53-c1

Competing interests

The authors declare no competing interests.

1. Ramos-Casals, M., Brito-Zerón, P., SisóAlmirall, A., Bosch, X. \& Tzioufas, A. G. Topical and systemic medications for the treatment of primary Sjögren's syndrome. Nat. Rev. Rheumatol. 8, 399-411 (2012).

2. Harbour, R. \& Miller, J. A new system for grading recommendations in evidence based guidelines. BMJ 323, 334-336 (2001).

3. Fox, R. I. Sjögren's syndrome. Lancet $\mathbf{3 6 6}$, 321-331 (2005).

4. Kassan, S. S. \& Moutsopoulos, H. M. Clinical manifestations and early diagnosis of Sjögren syndrome. Arch. Intern. Med. 164, 1275-1284 (2004).

5. Ramos-Casals, M., Tzioufas, A., Stone, J., Sisó, A. \& Bosch X. Treatment of primary Sjögren syndrome: a systematic review. JAMA 304, 452-460 (2010).

6. Roberts, C. W, Carniglia, P. E. \& Brazzo, B. G. Comparison of topical cyclosporine, punctal occlusion, and a combination for the treatment of dry eye. Cornea 26, 805-809 (2007).

7. Khurana, A. K., Moudgil, S. S., Ahluwalia, B. K. \& Parmar, I. P. Study of tear film flow and stability in chronic dacryocystitis. Acta Ophthalmol. (Copenh.) 65, 300-302 (1987).

8. Zengin, N. The effect of dacryocystorhinostomy on tear film flow and stability in patients with chronic dacryocystitis. Acta Ophthalmol. (Copenh.) 71, 714-716 (1993).

9. Yen, M. T., Pflugfelder, S. C. \& Feuer, W. J. The effect of punctal occlusion on tear production, tear clearance, and ocular surface sensation in normal subjects. Am. J. Ophthalmol. 131, 314-323 (2001).

10. Geldis, J. R. \& Nichols, J. J. The impact of punctal occlusion on soft contact lens wearing comfort and the tear film. Eye Contact Lens 34, 261-265 (2008). 